\title{
Introduction générale
}

\author{
Par François BOULOT, \\ Chef du Service RNE - EDF \\ Michel COUDRAY, \\ Directeur Technique - Framatome
}

René VIDAL,

Direction - IRDI - CEA

\section{Les motivations de la $R$ et $D$}

En 1969, la France adopte la filière à uranium enrichi et à eau ordinaire. Ce choix est alors dicté par des considérations d'ordre économique et industriel.

Ce faisant, l'industrie française accède au marché de la technologie des réacteurs nucléaires les plus répandus dans le monde et dont la part n'a d'ailleurs cessé de croître depuis lors. En 1974, la crise pétrolière mondiale conduit la France à engager un programme électronucléaire de grande envergure, exclusivement basé sur les réacteurs à eau ordinaire sous pression (REP) et caractérisé par la stratégie des paliers et contrats programmes adoptée par EDF

- 6 tranches de pré-série à Fessenheim et Bugey,

- 18 tranches de $900 \mathrm{MWe}$ du contrat programme CP1,

- 10 tranches de $900 \mathrm{MWe}$ du contrat programme CP2,

- 8 tranches de $1300 \mathrm{MWe}$ de la série P4,

- 12 tranches de $1300 \mathrm{MWe}$ de la série P'4.

Le nouveau palier N4 d'une puissance de $1400 \mathrm{MW}$ dont 2 tranches sont en construction, est l'aboutissement du processus de francisation complète de la filière menée depuis 1975 par Framatome, en liaison avec EDF et le CEA.

\section{- Assimilation et adaptation de la licence aux conditions françaises}

L'utilisation dans les meilleures conditions de la licence Westinghouse supposait de bien assimiler la technique qu'elle contenait et de l'adapter au contexte technique et réglementaire français.

Il a fallu tenir compte des conditions prévalant en France conditions techniques (fréquence du réseau à $50 \mathrm{~Hz}$, utilisation de matériaux et d'alliages différents), industrielles (fournisseurs et procédés de fabrication), réglementaires (sûreté, appareils à pression).

L'expérience nucléaire française du CEA, de Framatome et de EDF a permis de dominer rapidement les divers aspects des solutions mises en œuvre et d'aboutir à une maîtrise complète.

\section{- Amélioration des performances}

Le bon déroulement du programme électro-nucléaire conduisait à ce que le parc REP assurât déjà la majeure partie de la production d'électricité. II était alors devenu nécessaire de faire participer les tranches REP aux variations de la demande, en accroissant leur souplesse de fonctionnement alors que fondamentalement ces installations ont été conçues pour fonctionner en base.

Tout un programme de $R$ et $D$ a été engagé essentiellement à partir de 1975 pour adapter le réacteur à un fonctionnement en suivi de charge et en téléréglage ainsi que pour vérifier l'aptitude du combustible à supporter sans dommage les contraintes induites.

Un effort important de $\mathrm{R}$ et $\mathrm{D}$ a permis de comprendre et remédier aux difficultés et aux incidents rencontrés durant le fonctionnement et de surmonter les défauts de jeunesse, puis de proposer des améliorations comme celles du générateur de vapeur, l'un des composants essentiels des réacteurs REP.

\section{- La francisation}

L'expérience acquise au cours de la réalisation du programme a permis à la France de parfaire sa maîtrise de construction des REP. Dès lors au début des années 80 , il devenait possible d'établir de nouveaux liens avec Westinghouse sur une base d'égalité entre les deux partenaires. Ainsi une technologie française développée à l'origine sur la base de la technologie Westinghouse a permis progressivement d'accéder à l'indépendance technologique. La France est maintenant à même de proposer des produits français tant sur le marché national qu'à l'exportation comme les réacteurs dérivés du palier N4 ou les nouveaux combustibles type AFA.

\section{- La sûreté}

La responsabilité finale de la sûreté reposant sur l'exploitant, la préoccupation première d'EDF et de ses partenaires a toujours été d'assurer aux installations un très haut niveau de sûreté, souci déjà présent dès leur conception, notamment par la mise en œuvre de dispositifs visant à prévenir les incidents ou à en limiter les conséquences au cas où ils se produiraient. Mais la sûreté nucléaire ne peut rester figée: elle doit prendre en compte l'amélioration des connaissances sur les accidents obtenues à partir de programmes de $R$ et $D$ spécifiques (analyses probabilistes de sûreté par exemple) et incorporer les enseignements tirés du retour d'expérience d'exploitation. L'accident de Three Mile Island a amplifié ce besoin d'approfondissement dans le domaine de la sûreté opérationnelle. Cela s'est traduit par une amélioration des procédures accidentelles qui renforcent le niveau de sûreté des centrales REP.

\section{- Soutien à l'exploitant}

Dans un parc de centrales important, l'amélioration des conditions d'exploitation, tout en assurant un haut niveau de sûreté constitue une préoccupation majeure de l'exploitant. Cela se traduit par de nombreux travaux de $R$ et $D$ visant à :

- réduire les doses d'irradiation reçues par le personnel, - développer les outils de maintenance et optimiser la maintenance préventive,

- améliorer la fiabilité de certains matériels notamment sur la partie secondaire, source d'indisponibilités fortuites, beaucoup plus importante que celles liées au primaire,

- mieux cerner l'importance des facteurs humains dans l'exploitation des tranches,

- améliorer l'interface homme-machine en salle de commande par le développement des techniques d'informatisation pour la présentation des informations et la transmission des commandes,

\section{- Réduction du coût du kWh}

La réduction du coût du $\mathrm{kWh}$ est recherchée à travers la diminution du coût des investissements, du coût d'exploi- 
tation et du coût du cycle du combustible, qui constituent les trois composantes du coût du kWh.

La réduction du coût des investissements s'obtient par une amélioration de la conception des composants (GV, pompe, etc.), d'une part, en améliorant leurs performances grâce à la réduction des incertitudes apportées par les progrès effectués dans les différentes disciplines (neutronique, thermohydraulique, mécanique, métallurgie...), d'autre part, en augmentant leur fiabilité.

La réduction du coût d'exploitation implique plusieurs voies d'amélioration :

- amélioration de la disponibilité, par une réduction de la durée des arrêts programmés et par une diminution, en nombre et en durée, des arrêts non prévus,

- amélioration de la souplesse de fonctionnement des tranches: possibilité de faire varier les longueurs de campagne pour faciliter le placement des arrêts en période optimale et capacité des tranches à s'adapter aux besoins du réseau (suivi de charge et téléréglage),

- amélioration de la maintenance, par une optimisation des opérations d'entretien, par une amélioration des moyens de surveillance et de contrôle,

- amélioration de la gestion des déchets produits par les centrales, par le développement de procédés de conditionnement et d'élimination plus performants.

La réduction du coût du cycle du combustible, vu de la la centrale, met en jeu l'assemblage combustibe et son utilisation dans le réacteur. La réduction du coût du cycle passe donc par la recherche d'une augmentation des performances du combustible, par la meilleure utilisation en réacteur (gestion par $1 / 4$ de cœur au lieu de $1 / 3$ de cœur), par le recyclage du plutonium et de l'uranium de retraitement.

\section{- Préparation du long terme}

Cette dernière motivation de la $R$ et $D$ apparaît alors que se termine la phase de transformation du parc de production d'électricité par la substitution des tranches nucléaires aux tranches thermiques classiques. II s'agit désormais de préparer le parc du début du siècle prochain.

L'investigation se fait essentiellement suivant deux voies: - celle visant à prolonger, dans des conditions de sûreté requises, la durée de vie des tranches actuellement en service ou qui vont l'être prochainement, ce qui nécessitera des actions de $R$ et $D$ afin de mieux connaître les mécanismes de vieillissement des matériels, de mieux les surveiller et de prévoir des moyens d'entretien, de réparation correspondants,

- celle ayant pour but de rechercher de nouvelles installations de production thermique pour compléter le parc actuel et plus tard pour le remplacer, ce qui engendrera des actions de $R$ et $D$ sur la conception de ces moyens qui devront intégrer les progrès technologiques et les progrès de méthodes dans les différentes disciplines, et sur de nouveaux concepts de réacteur tels que les réacteurs à réseaux serrés ou à variation de spectre.

Cette préparation du long terme se fera en visant des gains significatifs sur les performances économiques et la sûreté.

\section{Les résultats obtenus}

Tous ces efforts développés tant sur l'outil de production que sur le combustible permettent aujourd'hui d'obtenir, en France, une électricité à plus de $70 \%$ d'origine nucléaire. Depuis 1977, 46 réacteurs à eau sous pression ont été couplés au réseau. Les performances des REP $900 \mathrm{MW}$ se sont avérées nettement supérieures aux prévisions initiales :

\section{L'EFFORT FINANCIER}

Les dépenses engagées par le CEA, EDF et Framatome, concernant les activités de Recherche et Développement sur la chaudière nucléaire - en conception, fabrication, exploitation et maintenance - et sur le combustible - hors enrichissement et retraitement - s'élèvent à un montant cumulé, depuis 1975 jusqu'en 1987, de l'ordre d'une douzaine de millards de francs courants.

Les prévisions budgétaires pour 1988 sont de 1,4 milliard de francs, dont la répartition est approximativement la suivante: 700 millions pour la chaudière, 400 millions pour le combustible et 300 millions sur les aspects spécifiques de sûreté.

A ces sommes, s'ajoutent les dépenses de Recherche et Développement prises en charge par les différents fabricants de composants, tels qu'Alsthom (concepteur de la turbine Arabelle).

Bien que le programme d'équipement se soit nettement ralenti, l'exploitation $d u$ parc actuel a besoin d'un support d'actions de $R$ et $D$ pour maintenir les qualités de la conception initiale, pour confirmer les performances de sûreté, de disponibilité d'aujourd'hui, pour améliorer le combustible et en particulier l'utilisation de la matière fissile et pour répondre aux problèmes qui ne manqueront pas de se poser au cours de la vie des tranches.

Ce nouveau paysage conduira dans le futur proche à une évolution progressive du contenu de la RechercheDéveloppement sur les centrales REP.

- leur disponibilité se situe au-delà de $80 \%$ au lieu de $70 \%$ prévu,

- leur souplesse d'exploitation remarquable analogue à celle présentée par le thermique classique permet de les faire participer aux actions de réglage de la puissance appelés sur le réseau,

- les doses intégrées par le personnel d'exploitation se situent à un faible niveau, comparé à celui des réacteurs exploités à l'étranger.

Ces efforts se sont également traduits par la francisation complète de la filière où les industriels sont devenus maîtres de leurs produits avec la mise au point des REP de type N4, les nouveaux types de combustibles comme l'AFA.

Egalement, en corollaire, une réglementation et des procédures ont été élaborées et mises en place, tant dans le domaine de la fabrication des matériels et de la construction que de l'exploitation et de la sûreté. Cela a permis de développer une organisation de la qualité performante et en amélioration constante.

Ainsi, forte de la compétence qu'elle a su se donner avec le programme national, l'industrie nucléaire française se trouve présente sur les marchés internationaux des centrales (Belgique, Afrique du Sud, Corée, Chine), sur celui du combustible (Suède, Allemagne, Belgique), celui des composants (Royaume-Uni) et celui des services (Espagne, USA).

\section{L'organisation de la $\mathbf{R}$ et $\mathbf{D}$}

\subsection{Les partenaires français}

L'organisation d'ensemble de la $R$ et $D$ en France résulte de l'organisation industrielle mise en place pour la réalisation du programme électronucléaire, à savoir :

- Framatome : concepteur, constructeur et vendeur de 


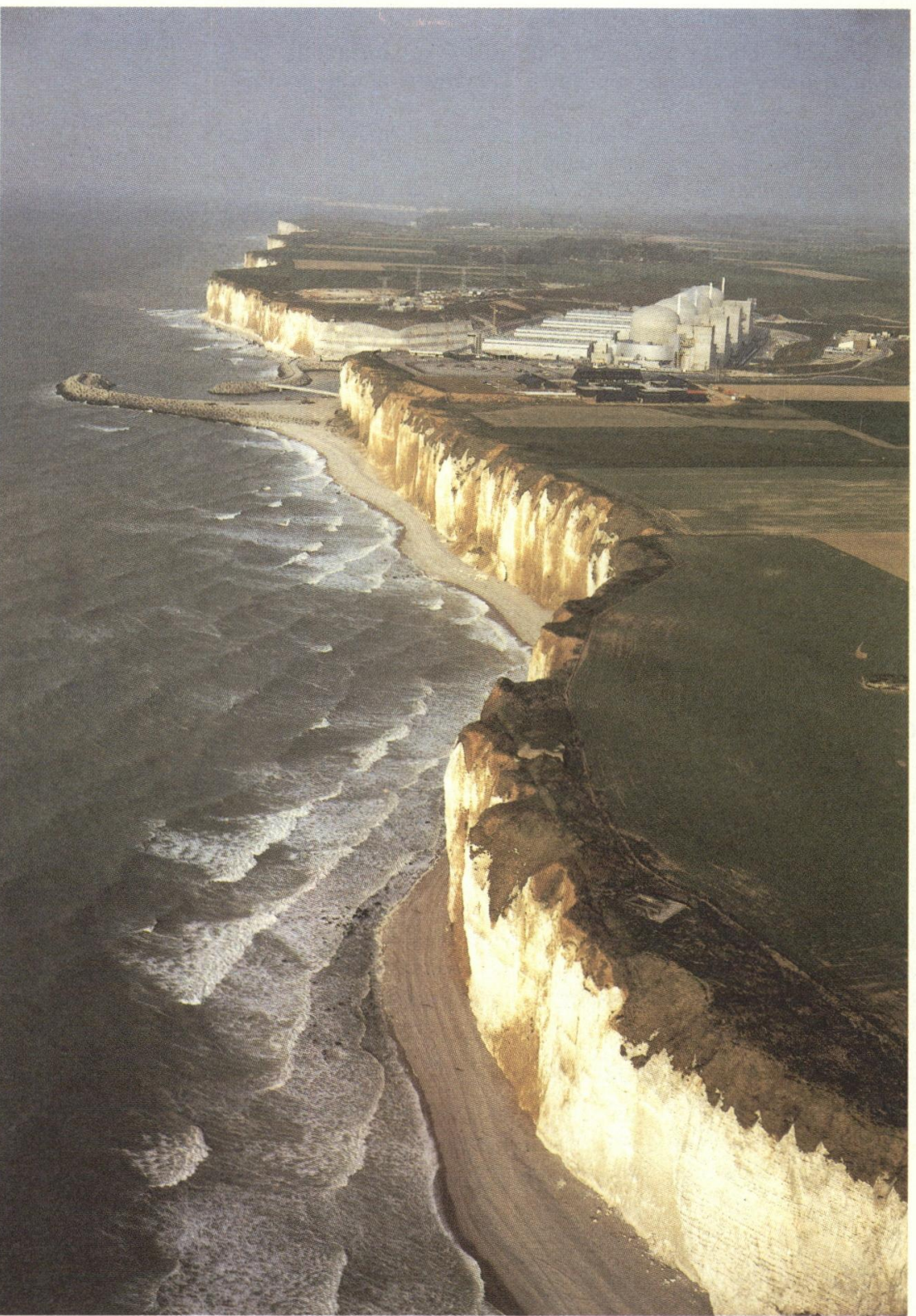

chaudières et îlots nucléaires, de combustibles et de services associés,

- EDF : propriétaire et exploitant des centrales, qui joue également le rôle d'architecte industriel et de concepteur d'ensemble de l'installation,

- CEA : organisme de $R$ et $D$, chargé notamment dans le domaine nucléaire de proposer des innovations et de forger les connaissances nécessaires à l'industrialisation des technologies.

La $R$ et $D$ à court ou moyen terme est exécutée selon les cas, chez l'un ou l'autre des partenaires suivant sa spécificité :

EDF prend en charge les études de gestion et d'optimisation du parc, les études liées à l'exploitation et au fonctionnement des tranches ainsi que les études de sûreté relatives à l'exploitation. En outre, EDF définit ses besoins futurs qui seront pris en compte dans l'établissement des programmes de $R$ et $D$.

Framatome a en charge les études de conception, de fabrication, d'ingénierie, et d'avant-projets de nouveaux concepts de réacteur et de nouveaux combustibles, ainsi que de nouveaux produits et services nucléaires destinés aux centrales existantes.
Le CEA apporte son concours aux études de systèmes et aux problèmes spécifiques posés par EDF ou Framatome. II assure également la majeure partie de la RetD concernant la physique du cœur, la technologie du combustible et la sûreté.

Cette énumération de tâches respectives n'a pour but que de donner des axes préférentiels de chacun des organismes.

En effet, en vue d'assurer un maximum de cohérence dans l'établissement des programmes de $R$ et $D$ et d'efficacité dans leur réalisation, est apparue très tôt la nécessité de mettre en place une étroite collaboration entre les trois principaux partenaires, concrétisée par une série d'accords qui les lient entre eux.

\subsection{Les accords nationaux}

Ceux-ci définissent le cadre contractuel pour engager en commun des actions de $R$ et $D$ entre deux ou trois partenaires. Ces accords reposent sur des comités qui en assurent la gestion: définition des programmes, répartition des tâches, engagement financier, contrôle du déroulement de l'action, etc. 
Parmi ces accords, on citera :

a) l'accord tripartite CEA-EDF-Framatome organisant la coopération dans les domaines de la chaudière et du combustible,

b) l'accord bipartite CEA-Framatome concernant la chaudière nucléaire et le combustible,

c) l'accord bipartite CEA-EDF ayant pour but de mieux tirer profit des enseignements issus de l'expérience d'exploitation des centrales,

d) la commission mixte CEA-EDF pour la sûreté des REP.

Divers autres accords spécifiques ayant un caractère de $R$ et $D$ plus ou moins marqué ont été également conclus entre un ou plusieurs des trois partenaires et des organismes tiers (Cogema, Intercontrôle, Zircotube, etc.).

\section{La coopération internationale}

Au niveau international, on peut constater une tendance générale à la recherche de collaboration en matière de $R$ et $D$, le coût de la $R$ et $D$ étant suffisamment important pour être incitatif en ce sens.

\subsection{L'accord quadripartite}

Parmi tous les accords conclus avec un partenaire étranger, il faut faire une place à part à l'accord quadripartite CEA-EDF-Framatome-Westinghouse.

Cet accord a été signé en novembre 1976, il a été renouvelé en novembre 1982 et prolongé en janvier 1988 pour une durée de cinq ans.

Ayant au départ été orienté pour faciliter l'assimilation de la technologie des REP par les partenaires français, l'accent a de plus en plus été mis au cours du temps, sur la résolution des problèmes rencontrés en exploitation. A l'avenir, l'objectif sera moins de réaliser de la $R$ et $D$ que d'échanger des informations sur l'expérience d'exploitation des centrales.

\subsection{Accords généraux d'échanges d'information}

Un certain nombre d'accords bi-latéraux, surtout dans le domaine de la sûreté, ont été conclus pour permettre des échanges d'informations sur les programmes de $R$ et $D$, notamment avec l'Allemagne Fédérale, les Etats-Unis, le Japon.

\subsection{Participation étrangères à des programmes français}

La valeur de la $R$ et $D$ française a été reconnue par de nombreux contrats d'études passés à un ou plusieurs des partenaires français (et parfois même eux ensembles) par des organismes comme les Communautés Européennes (sûreté notamment) ou l'EPRI (Groupement des Producteurs d'Electricité Américains) dans des domaines aussi variés que le combustible ou le générateur de vapeur.

Parfois même cette participation se concrétise sous la forme de programmes internationaux bâtis autour de programmes d'origine française tels que:

- CLOTAIRE (qualification des écoulements dans les générateurs de vapeur) avec participation d'EPRI, de Westinghouse, du Japon, de la Grande-Bretagne, du Canada, et de I'Italie.

- BETHSY (boucle système pour la sûreté des REP) avec pour certains essais, participation des Etats-Unis, du Japon et de l'Allemagne Fédérale.

- PHEBUS (étude des cœurs sévèrement dégradés) en collaboration avec le Japon et l'Allemagne Fédérale.

\subsection{Participation française à des programmes internationaux}

Dans ce cadre entrent essentiellement les programmes multinationaux mis en œuvre par le Centre Commun de Recherche de la CEE, tel que par exemple le programme LOBI pour l'analyse des conséquences d'une perte de réfrigérant. On peut mentionner également la participation des 3 partenaires français à l'important programme IPIRG (International Piping Integrity Research Group) lancé par la Nuclear Regulatory Commission Americaine pour l'étude du concept de fuite avant rupture. La validation de c'e concept permettrait de démontrer, dans certains cas, que la rupture brutale n'est pas à prendre en compte parmi les accidents de dimensionnement.

\section{ORGANISATION DU NUMERO}

Le parti adopté pour la structure de ce numéro consacré à la $R$ et $D$ sur les réacteurs à eau sous pression a été de faire le point des programmes en cours de développement par grand domaine technique avec un chapitre consacré à chacun d'eux.

On se limitera dans ce numéro à la chaudière nucléaire proprement dite. En effet vu l'importance de la $R$ et $D$ consacrée au combustible, celle-ci a été volontairement déconnectée et fera l'objet d'une série d'articles dans une nouvelle parution de la RGN. On ne parlera pas non plus de la partie classique même si la turbine Arabelle pour N4 constitue une innovation majeure pour laquelle une RetD conséquente a été réalisée.
Cinq grands chapitres ont donc été retenus :

- Le chapitre I présente les grands moyens d'essais nécessaires à la réalisation de cette $R$ et $D$.

- Le chapitre II traite de l'apport de la $R$ et $D$ dans la conception et la fabrication des différents composants.

- Le chapitre III a trait aux différentes actions menées en vue de mieux utiliser le parc de centrales existant.

- Le chapitre IV est consacré à la $R$ et $D$ relative à la sûreté.

- Le chapitre $V$ décrit les programmes d'actions en vue préparer le plus long terme.

Pour chaque grand chapitre, un texte d'introduction évoque les données principales et le contexte qui caractérisent le thème traité. 